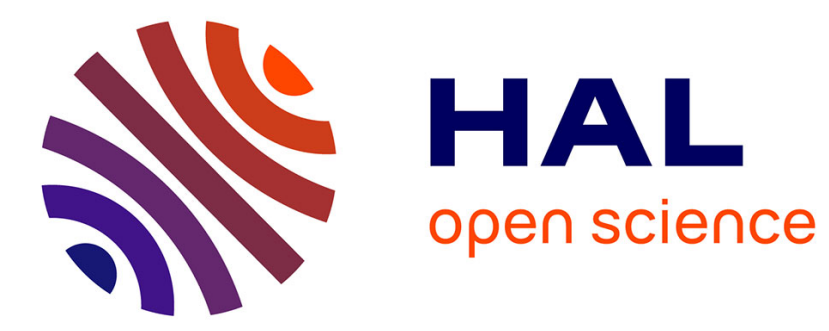

\title{
Wave propagation in two-dimensional periodic lattices
}

A. Srikantha Phani, J. Woodhouse, N. Fleck

\section{To cite this version:}

A. Srikantha Phani, J. Woodhouse, N. Fleck. Wave propagation in two-dimensional periodic lattices. Journal of the Acoustical Society of America, 2006, 119 (4), pp.1995-2005. 10.1121/1.2179748 . hal02273392

\section{HAL Id: hal-02273392 \\ https://hal.science/hal-02273392}

Submitted on 28 Aug 2019

HAL is a multi-disciplinary open access archive for the deposit and dissemination of scientific research documents, whether they are published or not. The documents may come from teaching and research institutions in France or abroad, or from public or private research centers.
L'archive ouverte pluridisciplinaire HAL, est destinée au dépôt et à la diffusion de documents scientifiques de niveau recherche, publiés ou non, émanant des établissements d'enseignement et de recherche français ou étrangers, des laboratoires publics ou privés. 


\title{
Wave propagation in two-dimensional periodic lattices
}

\author{
A. Srikantha Phani, J. Woodhouse, and N. A. Fleck ${ }^{\mathrm{a})}$ \\ Department of Engineering, Cambridge University, Trumpington Street, Cambridge CB2 1PZ, \\ United Kingdom
}

Plane wave propagation in infinite two-dimensional periodic lattices is investigated using Floquet-Bloch principles. Frequency bandgaps and spatial filtering phenomena are examined in four representative planar lattice topologies: hexagonal honeycomb, Kagomé lattice, triangular honeycomb, and the square honeycomb. These topologies exhibit dramatic differences in their long-wavelength deformation properties. Long-wavelength asymptotes to the dispersion curves based on homogenization theory are in good agreement with the numerical results for each of the four lattices. The slenderness ratio of the constituent beams of the lattice (or relative density) has a significant influence on the band structure. The techniques developed in this work can be used to design lattices with a desired band structure. The observed spatial filtering effects due to anisotropy at high frequencies (short wavelengths) of wave propagation are consistent with the lattice symmetries.

\section{INTRODUCTION}

Lattice materials are reticulated, cellular structures obtained by tessellating a unit cell comprising a few beams or bars (Gibson and Ashby, 1997). Engineering applications include sandwich beams, panels (Wicks and Hutchinson, 2001) and space trusses (Noor et al., 1978). The geometric periodicity of these materials governs their static and dynamic responses. The effective elastic properties of two-dimensional lattice materials and their dependence on relative density are well documented (Christensen, 2000; Gibson and Ashby, 1997; Torquato et al., 1998). Recent studies have also addressed the static elastic buckling and yielding phenomena of lattice materials (Hutchinson, 2004). Although considerable literature exists on their static properties, comparatively little is known about their wave propagation behavior.

The wave-bearing properties of a lattice are governed by its geometry. It has been observed by both physicists (e.g., Brillouin 1953; Kittel, 1962) and structural engineers (e.g., Langley et al., 1997; Mead, 1973, 1996) that wave motion in periodic structures, such as lattice materials, exhibits characteristic pass and stop bands. These pass bands and stop bands are frequency intervals over which wave motion can or cannot occur, respectively. There may be an opportunity to tailor lattice materials to achieve desired band gap characteristics, such that wave propagation is prevented in the specified frequency regimes. This behavior has direct application to photonic devices, and a systematic approach based on topology optimization procedures has been used to design photonic crystals with optimized bandgap properties (Sigmund and Jensen, 2003). They considered a square arrangement of inclusions of arbitrary shape, and they optimized the width of bandgap by varying the proportion of inclusion to the matrix, with the modulus and density of each phase held fixed. They observed the widest bandgap for the high contrast case, but

\footnotetext{
${ }^{a)}$ Electronic mail: naf1@eng.cam.ac.uk
}

limited their study to structures with square symmetry. Wave beaming also occurs in periodic structures: the direction of wave propagation at any given frequency is restricted to preferred directions (Langley et al., 1997; Ruzzene et al., 2003). Consequently, lattice materials behave as frequency and spatial filters.

Our objective in the present study is to explore wave propagation phenomena, such as bandgaps and wave directionality, in three regular honeycombs-hexagonal, square and triangular and in the semiregular Kagomé lattice. In all four geometries, the constituent beams are of uniform length $L$ and depth $d$, and two-dimensional (2D) prismatic topologies of unit thickness into the page are considered. The hexagonal and triangular honeycombs and the Kagomé lattice have isotropic in-plane effective properties, while the square honeycomb is strongly anisotropic (Gibson and Ashby, 1997; Torquato et al., 1998). The effective elastic properties of these microstructures are summarized in Table I. It is anticipated that the results based upon effective medium theory should agree with the asymptotes to the dispersion curves in the long-wavelength limit at zero frequency. Clearly, the four lattices have different long-wavelength deformation limits. It is of particular interest to know whether the finite frequency short-wavelength deformation behavior of the lattices show similar differences. To explore this, dispersion curves of the four lattices are computed using Floquet-Bloch principles. The Floquet-Bloch principles used in the present study have been widely employed in a number of research fields: in solid-state physics to investigate wave propagation in crystal structures, Bragg gratings and in photonic crystals (Brillouin, 1953; Kittel, 1962), and in mechanical systems such as stiffened panels to study their in-plane and out-of-plane vibration behavior (Mead, 1973, 1996).

The present study is structured as follows. FloquetBloch's principles are summarized in Sec. II in the context of lattice materials. The unit cell of each lattice is modeled as a network of beams using the finite element method, as de- 
TABLE I. Effective properties of the four lattice topologies: $E=$ Young's modulus of the solid material, $K^{*}$ and $G^{*}$ are the effective bulk modulus and shear modulus of the cellular solid, respectively. $\bar{\rho} \equiv \rho^{*} / \rho$ is relative density of the cellular solid, where $\rho$ and $\rho^{*}$ are the density of the solid material and lattice material, respectively. $d$ and $L$ are the thickness and length of the cell walls, respectively. $\lambda$ is the slenderness ratio defined as $\lambda=2 \sqrt{3} L / d$.

\begin{tabular}{|c|c|c|c|c|c|}
\hline Topology & Relative density $\bar{\rho}$ & $\begin{array}{c}\text { Relative bulk } \\
\text { modulus } \\
\bar{K}=\frac{K^{*}}{E}\end{array}$ & $\begin{array}{l}\text { Relative } \\
\text { shear } \\
\text { modulus } \\
\bar{G}=\frac{G^{*}}{E}\end{array}$ & Poisson's ratio $\nu^{*}$ & Isotropic \\
\hline $\begin{array}{l}\text { Triangular } \\
\text { honeycomb }\end{array}$ & $2 \sqrt{3}\left(\frac{d}{L}\right)=\frac{12}{\lambda}$ & $\frac{1}{4} \bar{\rho}$ & $\frac{1}{8} \bar{\rho}$ & $\frac{1}{3}$ & Yes \\
\hline $\begin{array}{l}\text { Hexagonal } \\
\text { honeycomb }\end{array}$ & $\frac{2}{\sqrt{3}}\left(\frac{d}{L}\right)=\frac{4}{\lambda}$ & $\frac{1}{4} \bar{\rho}$ & $\frac{3}{8} \bar{\rho}^{3}$ & 1 & Yes \\
\hline $\begin{array}{l}\text { Kagomé } \\
\text { lattice }\end{array}$ & $\sqrt{3}\left(\frac{d}{L}\right)=\frac{6}{\lambda}$ & $\frac{1}{4} \bar{\rho}$ & $\frac{1}{8} \bar{\rho}$ & $\frac{6-\bar{\rho}^{2}}{18+\bar{\rho}^{2}} \approx \frac{1}{3}$ & Yes \\
\hline $\begin{array}{l}\text { Square } \\
\text { honeycomb }\end{array}$ & $2\left(\frac{d}{L}\right)=\frac{4 \sqrt{3}}{\lambda}$ & $\frac{1}{4} \bar{\rho}$ & $\frac{1}{16} \bar{\rho}^{3}$ & 0 & No \\
\hline
\end{tabular}

scribed in Sec. III and the governing equations of motion are derived. The propagation of free harmonic waves is analysed in Sec. IV by applying Bloch's theorem to the governing equations of motion of the unit cell. A linear algebraic eigenvalue problem is formulated that involves the two components of the wave vector, called "phase constants," and the frequency of the plane wave. Dispersion surfaces are constructed by specifying the two phase constants and solving for the frequency. The dispersion surfaces for the four lattices and their long-wavelength asymptotes are discussed in Sec. V. Finally, the spatial filtering of waves due to anisotropic effects at high frequencies (corresponding to deformations of short wavelength) are considered with reference to the symmetries of the parent lattice.

\section{BLOCH'S THEOREM}

Before proceeding to Bloch's theorem, it is worth reviewing relevant concepts from solid state physics. The

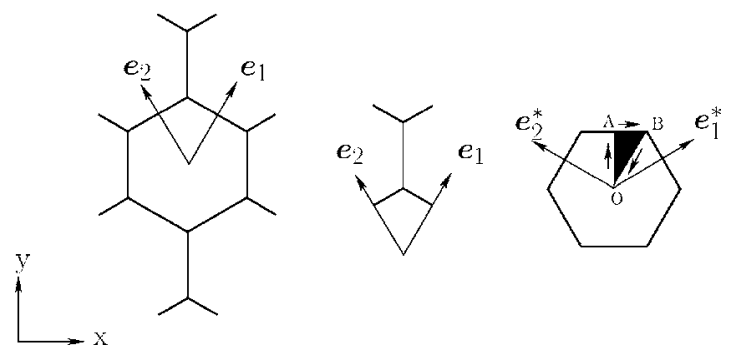

FIG. 1. Hexagonal honeycomb (left) with a selected primitive unit cell (middle) and the first Brillouin zone (right) in $k$ space. The basis vectors of the direct lattice $\left(\mathbf{e}_{i}\right)$ and the reciprocal lattice $\left(\mathbf{e}_{i}^{*}\right)$ are also shown. The points $\mathrm{O}, \mathrm{A}$, and $\mathrm{B}$ are as defined in Table III. joints of any lattice structure, such as the hexagonal honeycomb, can be envisioned as a collection of points, called lattice points, and these are associated with a set of basis vectors. The lattice point system together with the basis is usually referred to as a direct lattice.

Upon selecting a suitable unit cell, the entire direct lattice can be obtained by tesselating the unit cell along the basis vectors $\mathbf{e}_{i}$. Denote the lattice points in a unit cell by $\mathbf{r}_{j}$ : these will correspond to a subset of the nodes of the finite element model of the unit cell. Let $q\left(\mathbf{r}_{j}\right)$ denote the displacement of a lattice point in the reference unit cell. If a plane wave solution is admitted, then $q\left(\mathbf{r}_{j}\right)$ is of the form

$$
q\left(\mathbf{r}_{j}\right)=q_{j} e^{\left(i \omega t-\mathbf{k} \cdot \mathbf{r}_{j}\right)},
$$

where $q_{j}$ is the amplitude, $\omega$ is frequency in $\mathrm{rad} / \mathrm{s}$, and $\mathbf{k}$ is the wave vector of the plane wave. With reference to the chosen unit cell, let the integer pair $\left(n_{1}, n_{2}\right)$ identify any other cell obtained by $n_{1}$ translations along the $\mathbf{e}_{1}$ direction and $n_{2}$ translations along the $\mathbf{e}_{2}$ direction. The point in the cell $\left(n_{1}, n_{2}\right)$, corresponding to the $j$ th point in the reference

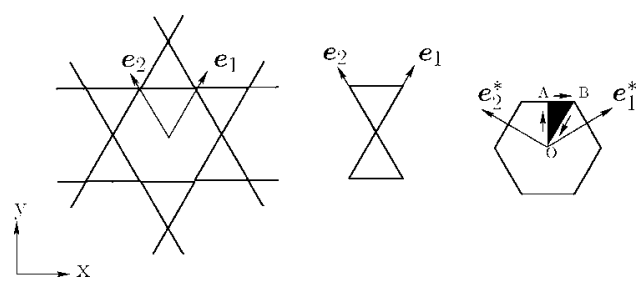

FIG. 2. Kagomé lattice (left) with selected primitive unit cell (middle) and the first Brillouin zone (right) in $k$ space. The basis vectors of the direct lattice $\left(\mathbf{e}_{i}\right)$ and the reciprocal lattice $\left(\mathbf{e}_{i}^{*}\right)$ are also shown. The points $\mathrm{O}, \mathrm{A}$ and $\mathrm{B}$ are as defined in Table III. 


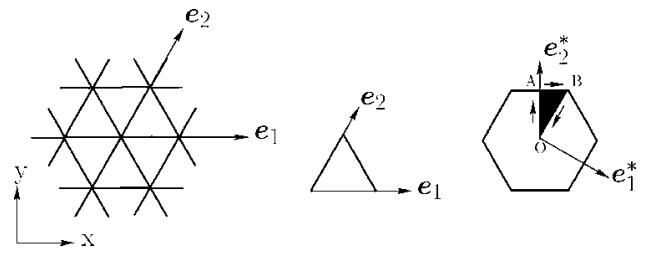

FIG. 3. Triangular honeycomb (left) with selected primitive unit cell (middle) and the first Brillouin zone (right) in $k$ space. The basis vectors of the direct lattice $\left(\mathbf{e}_{i}\right)$ and the reciprocal lattice $\left(\mathbf{e}_{i}^{*}\right)$ are also shown. The points $\mathrm{O}, \mathrm{A}$, and $\mathrm{B}$ are as defined in Table III.

unit cell, is denoted by the vector $\mathbf{r}=\mathbf{r}_{j}+n_{1} \mathbf{e}_{1}+n_{2} \mathbf{e}_{2}$. According to Bloch's theorem, the displacement at the $j$ th point in any cell identified by the integer pair $\left(n_{1}, n_{2}\right)$ in the direct lattice basis is given by

$$
q(\mathbf{r})=q\left(\mathbf{r}_{j}\right) e^{\mathbf{k} \cdot\left(\mathbf{r}-\mathbf{r}_{j}\right)}=q\left(\mathbf{r}_{j}\right) e^{\left(k_{1} n_{1}+k_{2} n_{2}\right)} .
$$

Here, $k_{1}=\delta_{1}+i \epsilon_{1}$ and $k_{2}=\delta_{2}+i \epsilon_{2}$ represent the components of the wave vector $\mathbf{k}$ along the $\mathbf{e}_{1}$ and $\mathbf{e}_{2}$ vectors, that is $k_{1}$ $=\mathbf{k} \cdot \mathbf{e}_{1}$ and $k_{2}=\mathbf{k} \cdot \mathbf{e}_{2}$. The real part $\delta$ and the imaginary part $\epsilon$ are called the attenuation and phase constants, respectively. The real part is a measure of the attenuation of a wave as it progresses from one unit cell to the next. For waves propagating without attenuation, the real part is zero and the components of the wave vector reduce to $k_{1}=i \epsilon_{1}$ and $k_{2}=i \epsilon_{2}$. The imaginary part or the phase constant is a measure of the phase change across one unit cell.

In simple terms, Bloch's theorem (or Floquet's principle in the case of one-dimensional periodic structures) states that for any structure with repetitive identical units, the change in complex wave amplitude across a unit cell, due to a propagating wave without attenuation, does not depend upon the location of the unit cell within the structure. By virtue of this theorem, one can understand wave propagation through the entire lattice by considering wave motion within a single unit cell. Bloch's theorem thus leads to enormous savings in the analysis of wave propagation in periodic structures.

It is convenient to define a reciprocal lattice in the wave vector space ( $k$ space), such that the basis vectors of the direct and reciprocal lattice satisfy

$$
\mathbf{e}_{i} \cdot \mathbf{e}_{j}^{*}=\delta_{i j},
$$

where $\mathbf{e}_{i}$ denote the basis vectors of the direct lattice and $\mathbf{e}_{j}^{*}$ denote the basis of reciprocal lattice, $\delta_{i j}$ is the Kronecker delta function. For a two-dimensional lattice, the subscripts $i$ and $j$ take the integer values 1 and 2 .

The wave vectors can be expressed in terms of the reciprocal lattice basis $\mathbf{e}_{i}^{*}$. Since the reciprocal lattice is also

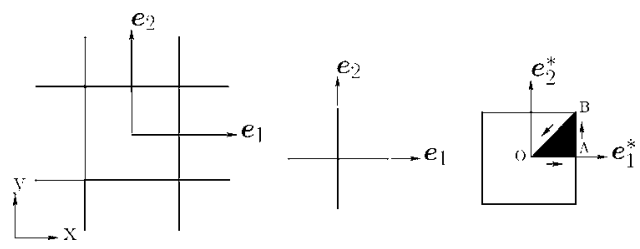

FIG. 4. Square honeycomb (left) with selected primitive unit cell (middle) and the first Brillouin zone (right) in $k$ space. The basis vectors of the direct lattice $\left(\mathbf{e}_{i}\right)$ and the reciprocal lattice $\left(\mathbf{e}_{i}^{*}\right)$ are also shown. The points $\mathrm{O}, \mathrm{A}$, and $\mathrm{B}$ are as defined in Table III.
TABLE II. Primitive translation vectors of the four lattices: $L$ denotes the length of each beam of the lattice; $\mathbf{i}$ and $\mathbf{j}$ are the Cartesian unit vectors in

\begin{tabular}{|c|c|c|}
\hline Topology & Direct lattice & Reciprocal lattice \\
\hline $\begin{array}{l}\text { Hexagonal } \\
\text { honeycomb }\end{array}$ & $\mathbf{e}_{1}=\sqrt{3} L\left(\frac{1}{2} \mathbf{i}+\frac{\sqrt{3}}{2} \mathbf{j}\right)$ & $\mathbf{e}_{1}^{*}=\frac{1}{\sqrt{3} L}\left(\mathbf{i}+\frac{1}{\sqrt{3}} \mathbf{j}\right)$ \\
\hline \multirow{3}{*}{$\begin{array}{l}\text { Kagomé } \\
\text { lattice }\end{array}$} & $\mathbf{e}_{2}=\sqrt{3} L\left(-\frac{1}{2} \mathbf{i}+\frac{\sqrt{3}}{2} \mathbf{j}\right)$ & $\mathbf{e}_{2}^{*}=\frac{1}{\sqrt{3} L}\left(-\mathbf{i}+\frac{1}{\sqrt{3}} \mathbf{j}\right.$ \\
\hline & $\mathbf{e}_{1}=2 L\left(\frac{1}{2} \mathbf{i}+\frac{\sqrt{3}}{2} \mathbf{j}\right)$ & $\mathbf{e}_{1}^{*}=\frac{1}{2 L}\left(\mathbf{i}+\frac{1}{\sqrt{3}} \mathbf{j}\right)$ \\
\hline & $\mathbf{e}_{2}=2 L\left(-\frac{1}{2} \mathbf{i}+\frac{\sqrt{3}}{2} \mathbf{j}\right)$ & $\mathbf{e}_{2}^{*}=\frac{1}{2 L}\left(-\mathbf{i}+\frac{1}{\sqrt{3}} \mathbf{j}\right)$ \\
\hline \multirow[t]{2}{*}{$\begin{array}{l}\text { Triangular } \\
\text { honeycomb }\end{array}$} & $\mathbf{e}_{1}=L \mathbf{i}$ & $\mathbf{e}_{1}^{*}=\frac{1}{L}\left(\mathbf{i}-\frac{1}{\sqrt{3}} \mathbf{j}\right)$ \\
\hline & $\mathbf{e}_{2}=L\left(\frac{1}{2} \mathbf{i}+\frac{\sqrt{3}}{2} \mathbf{j}\right)$ & $\mathbf{e}_{2}^{*}=\frac{1}{L}\left(\frac{2}{\sqrt{3}} \mathbf{j}\right)$ \\
\hline \multirow[t]{2}{*}{$\begin{array}{l}\text { Square } \\
\text { honeycomb }\end{array}$} & $\mathbf{e}_{1}=L \mathbf{i}$ & $\mathbf{e}_{1}^{*}=\frac{1}{L} \mathbf{i}$ \\
\hline & $\mathbf{e}_{2}=L \mathbf{j}$ & $\mathbf{e}_{2}^{*}=\frac{1}{L} \mathbf{j}$ \\
\hline
\end{tabular}
the $x-y$ plane. Note that $\mathbf{e}_{i} \cdot \mathbf{e}_{j}^{*}=\delta_{i j}$.

periodic, one can restrict the wave vectors to certain regions in the reciprocal lattice called Brillouin Zones (Brillouin, 1953). For computational efficiency, the wave vectors may be restricted to the edges of the irreducible part of the first Brillouin zone to explore bandgaps, since the band extrema almost always occur along the boundaries of the irreducible zone (Kittel, 1962). The first Brillouin zone is defined as a Wigner-Seitz or primitive unit cell of the reciprocal lattice, and it can be constructed as follows.

(1) Select any lattice point in the reciprocal lattice as the origin and connect it to neighboring points.

(2) Construct the perpendicular bisectors of these lines. The region of intersection is the first Brillouin zone.

The four lattices of interest in the present study are shown in Figs. 1-4 along with a choice of primitive unit cell, direct lattice translation vectors, and first Brillouin zone. The wave vectors are restricted to the edges of the irreducible first Brillouin Zone shown by the shaded region OAB. The basis vectors for the direct lattice and reciprocal lattice are tabulated in Table II and the Brillouin zone points are tabulated in Table III for each lattice. 
TABLE III. The irreducible first Brillouin zone points of the four lattices. The wave vectors are chosen along the locus $\mathrm{OAB}$; i.e.; along the edges of the irreducible part of the first Brillouin zone.

\begin{tabular}{lll}
\hline \hline \multicolumn{1}{c}{ Topology } & Cartesian basis & Reciprocal basis \\
\hline $\begin{array}{l}\text { Hexagonal } \\
\text { honeycomb }\end{array}$ & $\mathrm{O}=(0,0) ; \quad \mathrm{A}=\frac{1}{L}\left(0, \frac{1}{3}\right) ; \quad \mathrm{B}=\frac{1}{L}\left(\frac{1}{3 \sqrt{3}}, \frac{1}{3}\right)$ & $\mathrm{O}=(0,0) ; \quad \mathrm{A}=\left(\frac{1}{2}, \frac{1}{2}\right) ; \quad \mathrm{B}=\left(\frac{2}{3}, \frac{1}{3}\right)$ \\
$\begin{array}{l}\text { Kagomé } \\
\text { lattice }\end{array}$ & $\mathrm{O}=(0,0) ; \mathrm{A}=\frac{1}{L}\left(0, \frac{1}{3}\right) ; \quad \mathrm{B}=\frac{1}{L}\left(\frac{1}{3 \sqrt{3}}, \frac{1}{3}\right)$ & $\mathrm{O}=(0,0) ; \quad \mathrm{A}=\left(\frac{1}{2}, \frac{1}{2}\right) ; \quad \mathrm{B}=\left(\frac{2}{3}, \frac{1}{3}\right)$ \\
$\begin{array}{l}\text { Triangular } \\
\text { honeycomb }\end{array}$ & $\mathrm{O}=(0,0) ; \quad \mathrm{A}=\frac{1}{L}\left(0, \frac{1}{\sqrt{3}}\right) ; \quad \mathrm{B}=\frac{1}{L}\left(\frac{1}{3}, \frac{1}{\sqrt{3}}\right)$ & $\mathrm{O}=(0,0) ; \mathrm{A}=\left(0, \frac{1}{2}\right) ; \quad \mathrm{B}=\left(\frac{1}{3}, \frac{2}{3}\right)$ \\
$\begin{array}{l}\text { Square } \\
\text { honeycomb }\end{array}$ & $\mathrm{O}=(0,0) ; \quad \mathrm{A}=\frac{1}{L}\left(0, \frac{1}{2}\right) ; \quad \mathrm{B}=\frac{1}{L}\left(\frac{1}{2}, \frac{1}{2}\right)$ & $\mathrm{O}=(0,0) ; \quad \mathrm{A}=\left(0, \frac{1}{2}\right) ; \quad \mathrm{B}=\left(\frac{1}{2}, \frac{1}{2}\right)$
\end{tabular}

\section{FINITE ELEMENT MODELING OF THE UNIT CELL}

Each lattice is considered to be a rigid-jointed network of beams with no prestress. The unit cell is discretized into a network of Timoshenko beams. Each beam is assumed to have three degrees of freedom at each end: two translations $(u, v)$ in the $(x, y)$ plane and a rotation $\theta_{z}$ about the $z$ axis. The continuous variation of these displacements within a typical beam element, shown in Fig. 5, is approximated by

$$
\begin{aligned}
& u(x, t)=\sum_{r=1}^{6} a_{r}(x) q_{r}(t), \quad v(x, t)=\sum_{r=1}^{6} b_{r}(x) q_{r}(t), \\
& \theta_{z}(x, t)=\sum_{r=1}^{6} c_{r}(x) q_{r}(t),
\end{aligned}
$$

where $x$ is measured along the axis of the beam, and the degrees of freedom $q_{r}$ consist of the six nodal degrees of freedom $\left(u_{1}, v_{1}, \theta_{z 1}, u_{2}, v_{2}, \theta_{z 2}\right)$. The shape functions $a_{r}, b_{r}$, and $c_{r},(r=1 \cdots 6)$ for the six nodal displacements are given in Appendix A.

The kinetic and potential energies per unit thickness of the beam into the page are given by:

$$
T=\frac{1}{2} \int_{-L / 2}^{L / 2} \rho d \dot{u}^{2} d x+\frac{1}{2} \int_{-L / 2}^{L / 2} \rho d \dot{v}^{2} d x+\frac{1}{2} \int_{-L / 2}^{L / 2} \rho I_{z} \dot{\theta}_{z}^{2} d x
$$

$$
\begin{aligned}
U= & \frac{1}{2} \int_{-L / 2}^{L / 2} E d\left(\frac{d u}{d x}\right)^{2} d x+\frac{1}{2} \int_{-L / 2}^{L / 2} E I_{z}\left(\frac{d \theta_{z}}{d x}\right)^{2} d x \\
& +\frac{1}{2} \int_{-L / 2}^{L / 2} \kappa d G\left(\frac{d v}{d x}-\left(\theta_{z}\right)\right)^{2} d x,
\end{aligned}
$$

where $\rho$ is the density of the material used to make the lattice. $L$ and $I_{z}$ denote the length and second moment of area of the beam, respectively; and $\kappa$ denotes the shear correction factor used in Timoshenko beam theory (Weaver and Jonhston, 1987). Substitute Eq. (4) into Eq. (5) in order to obtain

$$
\begin{aligned}
T= & \frac{1}{2} \sum_{r=1}^{6} \sum_{s=1}^{6} \dot{q}_{r} \dot{q}_{s} \int_{-L / 2}^{L / 2}\left(\rho d a_{r} a_{s}+\rho d b_{r} b_{s}+\rho I_{z} c_{r} c_{s}\right) d x, \\
U= & \frac{1}{2} \sum_{r=1}^{6} \sum_{s=1}^{6} q_{r} q_{s} \int_{-L / 2}^{L / 2}\left[E d a_{r}^{\prime} a_{s}^{\prime}+E I_{z} b_{r}^{\prime \prime} b_{s}^{\prime \prime}+\kappa G d\left(b_{r}^{\prime}-c_{r}\right)\right. \\
& \left.\times\left(b_{s}^{\prime}-c_{s}\right)\right] d x,
\end{aligned}
$$

where the primes denote differentiation with respect to the axial coordinate $x$. The equations of motion are obtained by applying Hamilton's variational principle,

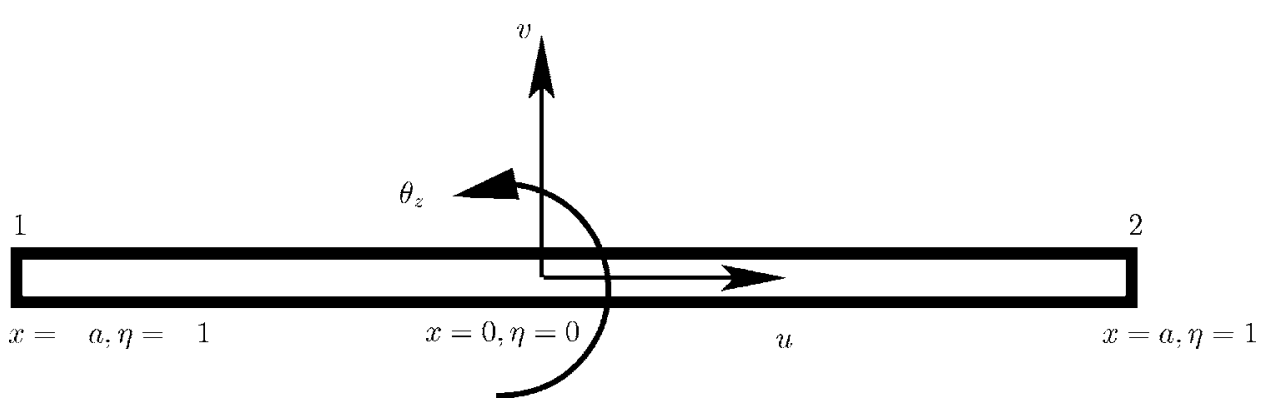

FIG. 5. Beam element with nodes numbered 1 and 2 . The three nodal degrees of freedom are shown together with the local element coordinate axes with origin located at the middle of the beam. The nondimensional coordinate is $\eta=x / a=2 x / L$, where $L$ is the length of the beam. 


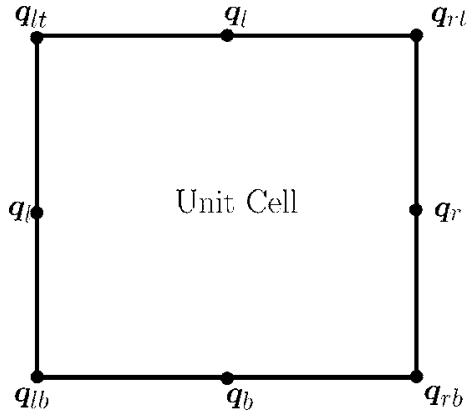

FIG. 6. A generic unit cell for a two-dimensional periodic structure, showing the degrees of freedom shared with the neighboring unit cells.

$$
\delta \int \mathcal{L} d t=0, \quad \mathcal{L}=T-U+W_{e},
$$

where $\delta$ denotes the first variation and $W_{e}$ denotes the work done by the external forces. Upon evaluating the first variation in Eq. (7), the Euler-Lagrangian equations of motion for the dynamics of the beam element are obtained as

$$
\frac{d}{d t}\left(\frac{\partial \mathcal{L}}{\partial \dot{q}_{r}}\right)-\frac{\partial \mathcal{L}}{\partial q_{r}}=f_{r},
$$

where $\mathcal{L}$ is the Lagrangian of the dynamical system as defined in Eq. (7) and $f_{r}$ is the force corresponding to the
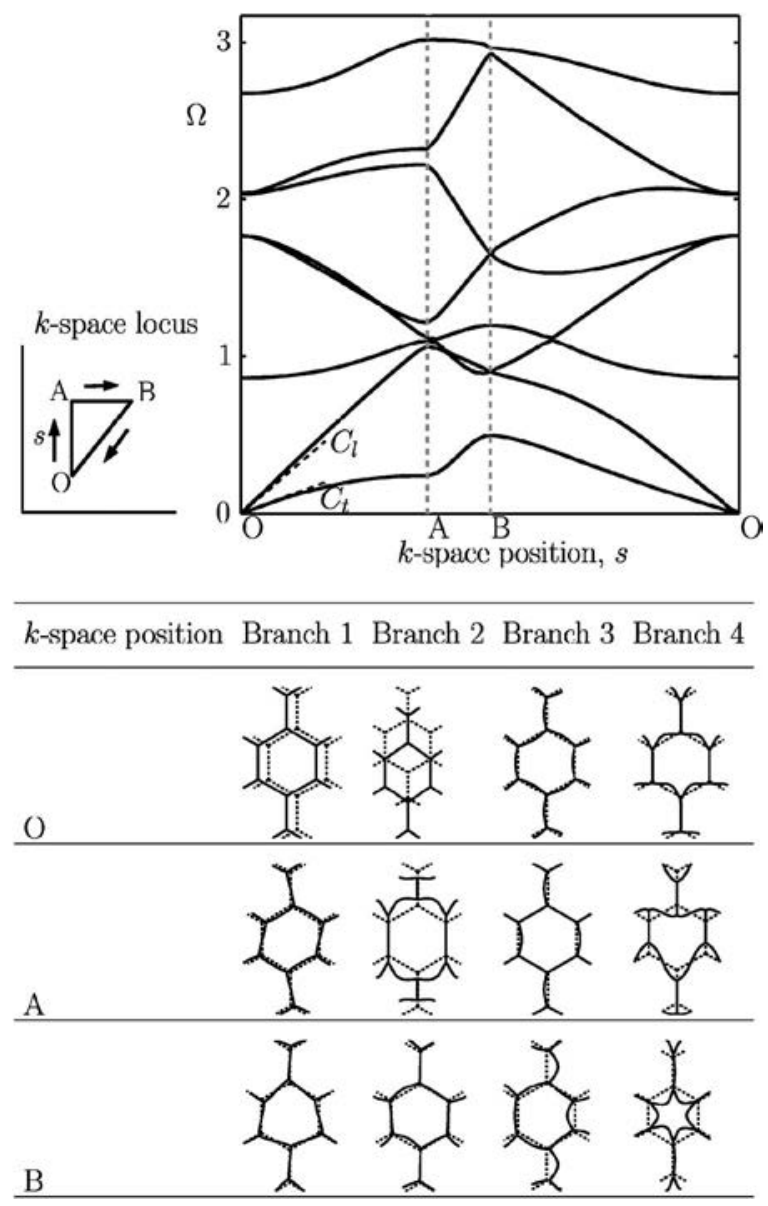

FIG. 7. Band structure of a hexagonal honeycomb with slenderness ratio equal to 10 . The eigenwaves of a typical cell are shown in tabular form. The three rows correspond to the three points $\mathrm{O}, \mathrm{A}$, and $\mathrm{B}$ in $k$ space, while the four columns correspond to the first four dispersion branches in ascending order. degree of freedom $q_{r}$. The above equations of motion can be written for each beam element and the assembled equation of the motion for the unit cell take the form

$$
\mathbf{M} \ddot{\mathbf{q}}+\mathbf{K q}=\mathbf{f},
$$

where the matrices $\mathbf{M}, \mathbf{K}$ denote the assembled global mass and stiffness matrix of the unit cell, respectively. The vectors $\mathbf{q}$, $\ddot{\mathbf{q}}$, and $\mathbf{f}$, respectively, denote the nodal displacements, accelerations, and forces. For any $j$ th node the nodal displacement vector is given by $\mathbf{q}_{j}=\left[u_{j} v_{j} \theta_{z j}\right]^{T}$.

\section{ANALYSIS OF FREE WAVE MOTION}

Having formulated the equations of motion of a unit cell, the propagation of planar harmonic waves at a radial frequency $\omega$ within the entire lattice can be investigated by invoking Bloch's theorem. The equations of motion in Eq. (9) follow as

$$
\left[-\omega^{2} \mathbf{M}+\mathbf{K}\right] \mathbf{q}=\mathbf{f} \text { or } \mathbf{D q}=\mathbf{f}, \quad \mathbf{D}=\left[-\omega^{2} \mathbf{M}+\mathbf{K}\right],
$$

where the dynamic stiffness $\mathbf{D}$ reduces to the static stiffness at zero frequency.

By virtue of Bloch's theorem, the following relationships between the displacements, $\mathbf{q}$, and forces, $\mathbf{f}$, are obtained:

$$
\begin{aligned}
& \mathbf{q}_{r}=e^{k_{1}} \mathbf{q}_{l}, \quad \mathbf{q}_{t}=e^{k_{2}} \mathbf{q}_{b}, \\
& \mathbf{q}_{r b}=e^{k_{1}} \mathbf{q}_{l b}, \quad \mathbf{q}_{r t}=e^{k_{1}+k_{2}} \mathbf{q}_{l b}, \quad \mathbf{q}_{l t}=e^{k_{2}} \mathbf{q}_{l b} \\
& \mathbf{f}_{r}=-e^{k_{1}} \mathbf{f}_{l}, \quad \mathbf{f}_{t}=-e^{k_{2}} \mathbf{f}_{b}, \\
& \mathbf{f}_{r t}+e^{k_{1}} \mathbf{f}_{l t}+e^{k_{2}} \mathbf{f}_{r b}+e^{k_{1}+k_{2}} \mathbf{f}_{l b}=0,
\end{aligned}
$$

where the subscripts $l, r, b, t$, and $i$, respectively, denote the displacements corresponding to the left, right, bottom, top, and internal nodes of a generic unit cell, as shown in Fig. 6. The displacements of the corner nodes are denoted by double subscripts: for example, $l b$ denotes the left bottom corner.

Using the above relationships one can define the following transformation:

$$
\begin{aligned}
& \mathbf{q}=\mathbf{T} \widetilde{\mathbf{q}}, \\
& \mathbf{T}=\left[\begin{array}{cccc}
\mathbf{I} & \mathbf{0} & \mathbf{0} & \mathbf{0} \\
\mathbf{I} e^{k_{1}} & \mathbf{0} & \mathbf{0} & \mathbf{0} \\
\mathbf{0} & \mathbf{I} & \mathbf{0} & \mathbf{0} \\
\mathbf{0} & \mathbf{I} e^{k_{2}} & \mathbf{0} & \mathbf{0} \\
\mathbf{0} & \mathbf{0} & \mathbf{I} & \mathbf{0} \\
\mathbf{0} & \mathbf{0} & \mathbf{I} e^{k_{1}} & \mathbf{0} \\
\mathbf{0} & \mathbf{0} & \mathbf{I} e^{k_{2}} & \mathbf{0} \\
\mathbf{0} & \mathbf{0} & \mathbf{I} e^{\left(k_{1}+k_{2}\right)} & \mathbf{0} \\
\mathbf{0} & \mathbf{0} & \mathbf{0} & \mathbf{I}
\end{array}\right], \quad \widetilde{\mathbf{q}}=\left[\begin{array}{c}
\mathbf{q}_{l} \\
\mathbf{q}_{b} \\
\mathbf{q}_{l b} \\
\mathbf{q}_{i}
\end{array}\right],
\end{aligned}
$$

where $\widetilde{\mathbf{q}}$ denote the displacements of the nodes in the Bloch reduced coordinates. Now, substitute the transformation given by Eq. (12) into the governing equations of motion in Eq. (10) and premultiply the resulting equation with $\mathbf{T}^{\mathrm{H}}$ to enforce force equilibrium (Langley, 1993). One obtains the 

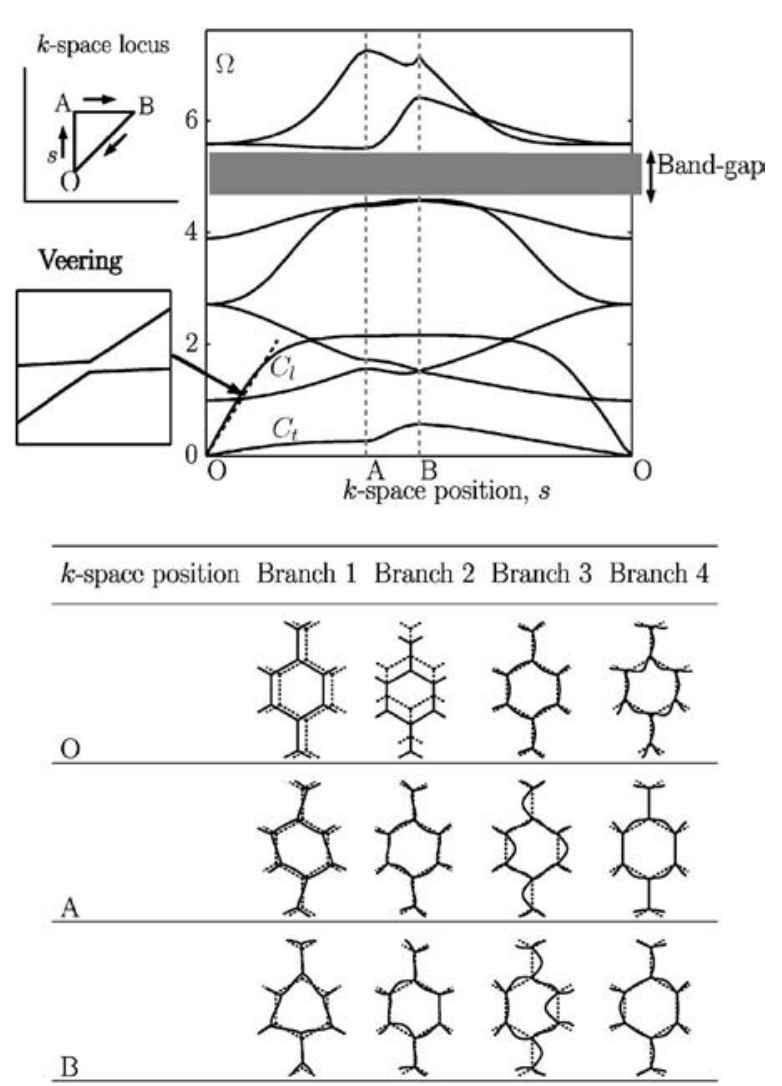

FIG. 8. Band structure of a hexagonal honeycomb with a slenderness ratio equal to 50. The eigenwaves of a typical cell are shown in tabular form. The three rows correspond to the three points $\mathrm{O}, \mathrm{A}$, and $\mathrm{B}$ in $k$ space, while the four columns correspond to the first four dispersion branches in ascending order.

following governing equations in the reduced coordinates:

$$
\widetilde{\mathbf{D}} \widetilde{\mathbf{q}}=\widetilde{\mathbf{f}}, \quad \widetilde{\mathbf{D}}=\mathbf{T}^{\mathrm{H}} \mathbf{D} \mathbf{T}, \quad \tilde{\mathbf{f}}=\mathbf{T}^{\mathrm{H}} \mathbf{f},
$$

where the superscript ${ }^{\mathrm{H}}$ denotes the Hermitian transpose. For a plane wave propagating without attenuation in the $x-y$ plane, the propagation constants along the $x$ and $y$ directions are $k_{1}=i \epsilon_{1}$ and $k_{2}=i \epsilon_{2}$. For free wave motion $(\mathbf{f}=0)$, the above equation can be written in the frequency domain to give the following eigenvalue problem:

$$
\widetilde{\mathbf{D}}\left(k_{1}, k_{2}, \omega\right) \widetilde{\mathbf{q}}=0 .
$$

Any triad $\left(k_{1}, k_{2}, \omega\right)$ obtained by solving the eigenvalue problem in Eq. (14) represents a plane wave propagating at frequency $\omega$.

In the eigenvalue problem defined by Eq. (14), there exist three unknowns: the two propagation constants $k_{1}, k_{2}$ that are complex, in general, and the frequency of wave propagation $\omega$ that is real, since the matrix $\widetilde{\mathbf{D}}$ in the eigenvalue problem is Hermitian. At least two of the three unknowns have to be specified to obtain the third. For wave motion without attenuation the propagation constants are purely imaginary of the form $k_{1}=i \epsilon_{1}$ and $k_{2}=i \epsilon_{2}$. In this case one obtains the frequencies of wave propagation as a solution to the linear algebraic eigenvalue problem defined in Eq. (14) for each pair of phase constants $\left(\epsilon_{1}, \epsilon_{2}\right)$. The solution is a surface, called the dispersion surface, in the $\omega-k_{1}-k_{2}$ coordinates. There exist as many surfaces as there are eigen-
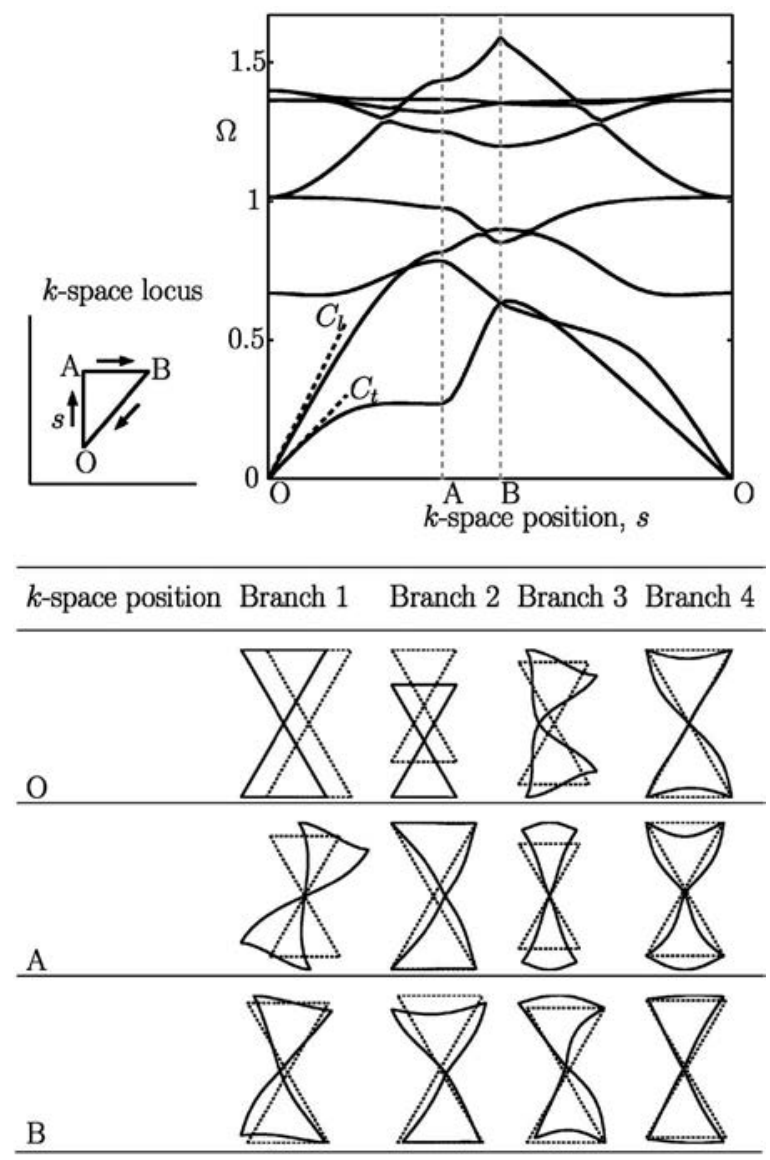

FIG. 9. Band structure of a Kagomé lattice with slenderness ratio equal to 10. The eigenwaves of a typical cell are shown in tabular form. The three rows correspond to the three points $\mathrm{O}, \mathrm{A}$, and $\mathrm{B}$ in $k$ space, while the four columns correspond to the first four dispersion branches in ascending order.

values of the problem in Eq. (14). If two surfaces do not overlap each other then there is a gap along the $\omega$ axis in which no wave motion occurs. This gap between dispersion surfaces is called the bandgap in the solid-state physics literature (Kittel, 1962) and the stop band in structural dynamics (Mead, 1973, 1996). For all frequencies on the phase constant surface, wave motion can occur and hence the frequency range occupied by these surfaces is a pass band. Furthermore, the normal to the phase constant surface at any point gives the Poynting vector or group velocity, and this indicates the speed and direction of energy flow.

\section{BAND STRUCTURE OF THE FOUR LATTICE TOPOLOGIES}

The computational procedure adopted to calculate the dispersion surfaces (band structure) for the four lattices is as follows.

(1) Select a primitive unit cell of the lattice.

(2) Construct the mass and stiffness matrices of the unit cell using the finite element technique described in Sec. III.

(3) Apply Bloch's principle to the equations of motion of the unit cell and form the eigenvalue problem in Eq. (14).

(4) Specify the phase constants $\left(\epsilon_{1}, \epsilon_{2}\right)$ by restricting the wave vector to the edges of the irreducible part of the first Brillouin zone. 

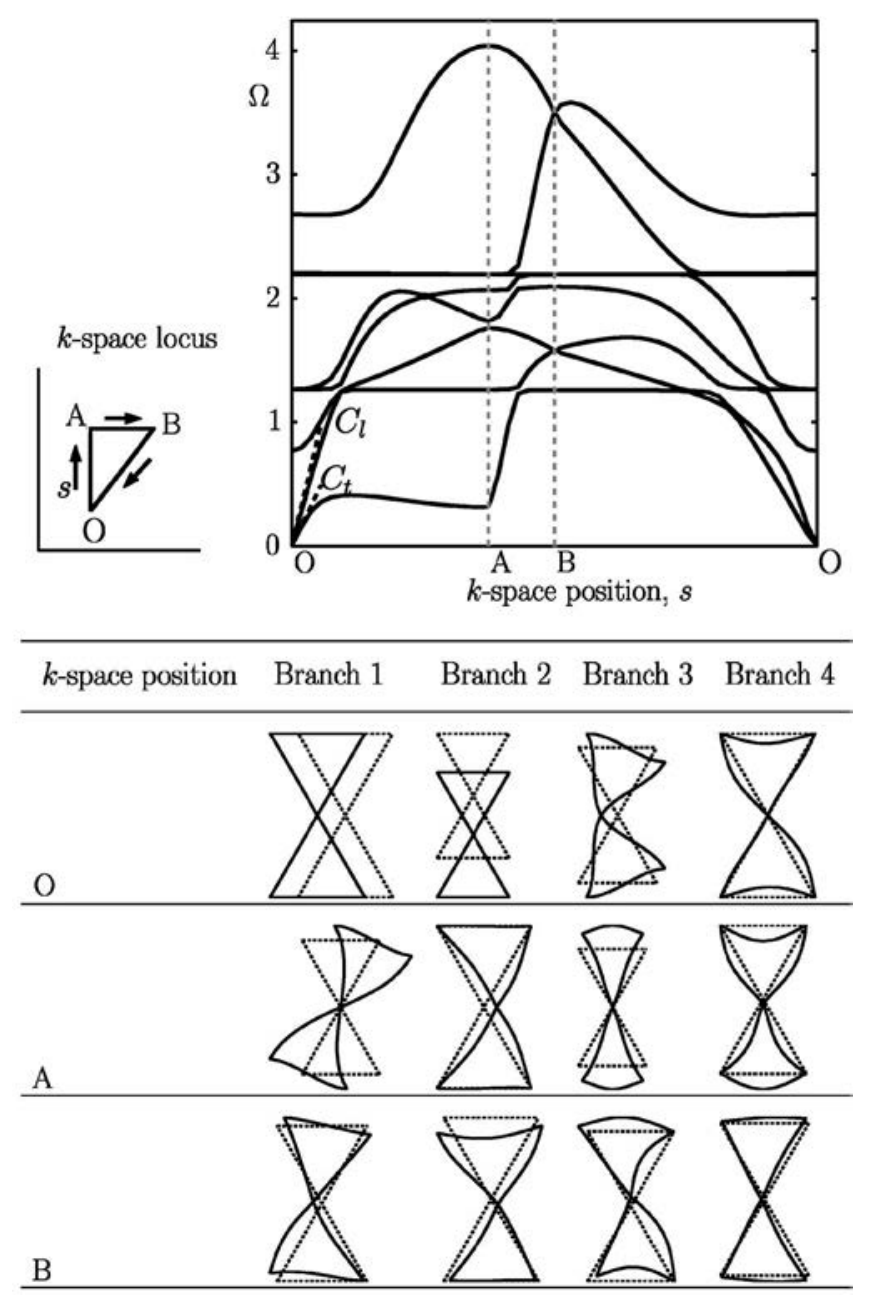

FIG. 10. Band structure of a Kagomé lattice with a slenderness ratio equal to 50. The eigenwaves of a typical cell are shown in tabular form. The three rows correspond to the three points $\mathrm{O}, \mathrm{A}$, and $\mathrm{B}$ in $k$ space, while the four columns correspond to the first four dispersion branches in ascending order.

(5) Solve the resulting linear algebraic eigenvalue problem in Eq. (14) for the wave propagation frequencies.

The four lattices are shown in Figs. 1-4 along with the choice of primitive unit cell, direct lattice translation vectors, and the first Brillouin zone. To explore the bandgaps it is sufficient to choose wave vectors along the edges of the first irreducible Brillouin zone (Kittel, 1962). Instead of solving the eigenvalue problem in Eq. (14) for each pair $\left(\epsilon_{1}, \epsilon_{2}\right)$ over the shaded region $\mathrm{OAB}$ in the first Brillouin zone, one need only explore the edges of the triangle $\mathrm{OAB}$. The parameter $s$ is introduced as the arclength along the perimeter $\mathrm{OABO}$ of the shaded region $\mathrm{OAB}$ in the first Brillouin zone. It is a scalar pathlength parameter in $k$ space and is used to denote the location of any point on the perimeter. Thus, the extremes of the frequency on a three-dimensional dispersion surface can be represented by a two-dimensional dispersion curve with the wave vector as the abscissa and the frequency as the ordinate. The bandgaps now correspond to regions along the ordinate wherein no dispersion branch is present. Within this frequency band wave motion cannot occur, and hence these are stop bands. In contrast, the frequency values for which there is at least one dispersion curve correspond to the pass
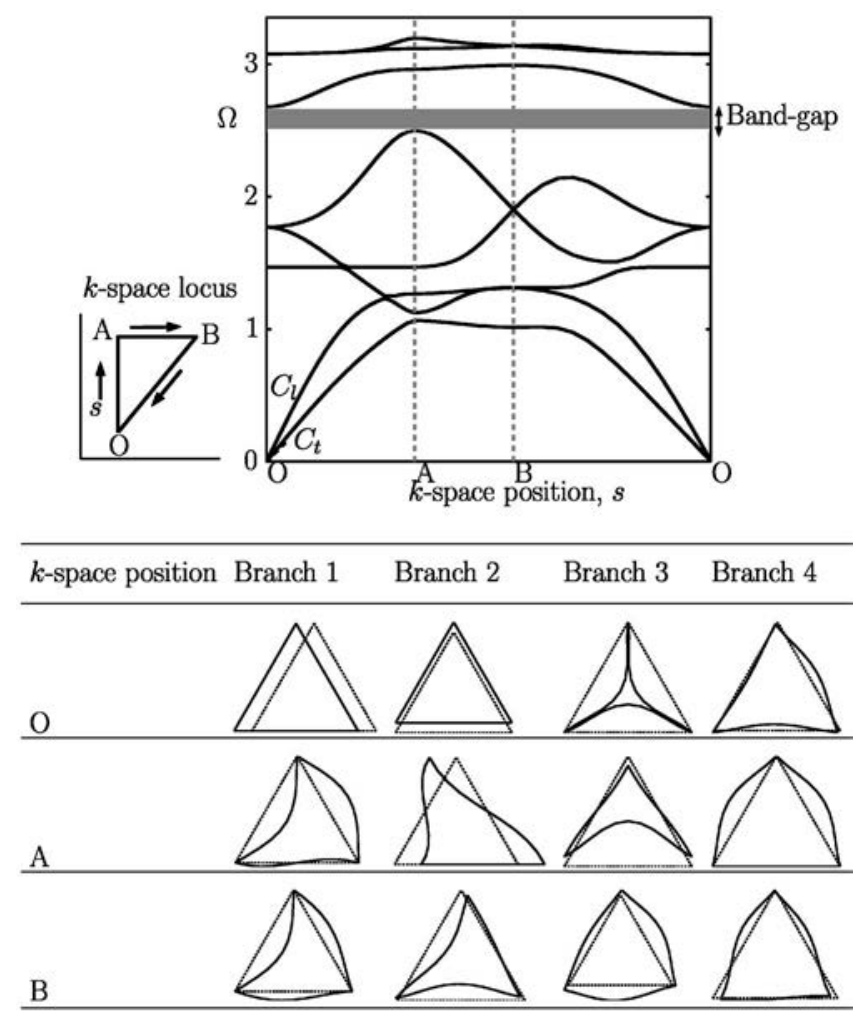

FIG. 11. Band structure of a triangular honeycomb with a slenderness ratio equal to 10. The eigenwaves of a typical cell are shown in tabular form. The three rows correspond to the three points $\mathrm{O}, \mathrm{A}$, and $\mathrm{B}$ in $k$ space, while the four columns correspond to the first four dispersion branches in ascending order.

bands. The long-wavelength limit corresponds to the origin, as denoted by point $\mathrm{O}$ in the first Brillouin zone.

The band structure of each lattice is computed by solving the eigenvalue problem in Eq. (14) for wave vectors along the closed locus O-A-B-O in $k$ space. Results are presented for the two extremes of the slenderness ratio $(\lambda$ $=2 \sqrt{3} L / d$ ) equal to 10 and 50 . Recall that the relative density scales directly with the slenderness ratio, and the scaling factor for each geometry is summarized in Table I. For each topology, the band structure of the lattice with slenderness ratio equal to 20 was found to be similar to that of a lattice with slenderness ratio of 10 , and so these plots are omitted in the interest of brevity. We consider in turn the hexagonal honeycomb, Kagomé lattice, triangular honeycomb, and finally the square honeycomb.

\section{A. Hexagonal honeycomb}

The dispersion curves for slenderness ratios of 10 and 50 are shown in Figs. 7 and 8. In these plots a nondimensional wave propagation frequency is plotted on the vertical axis as a function of the wave vector locus O-A-B-O along the edges of the irreducible first Brillouin zone, using the arclength parameter $s$ in $k$ space. A convenient nondimensional frequency $\Omega$ is defined as 

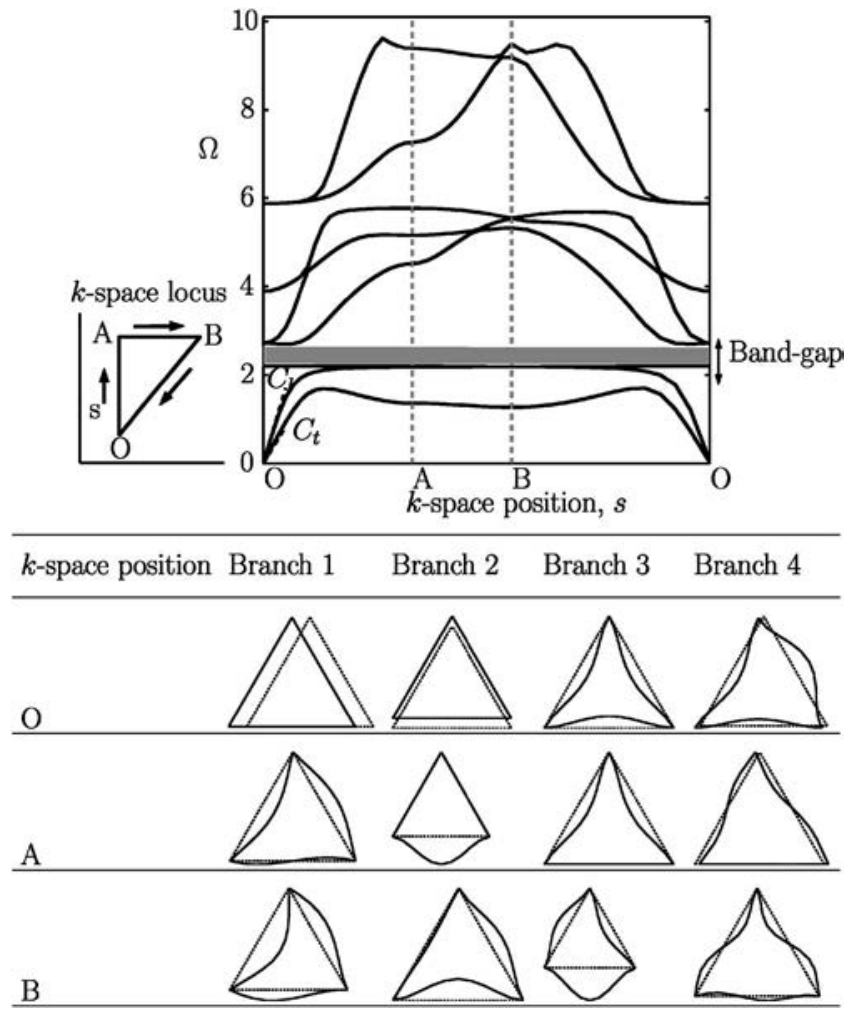

FIG. 12. Band structure of a triangular honeycomb with a slenderness ratio equal to 50. The eigenwaves of a typical cell are shown in tabular form. The three rows correspond to the three points $\mathrm{O}, \mathrm{A}$, and $\mathrm{B}$ in $k$ space, while the four columns correspond to the first four dispersion branches in ascending order.

$$
\Omega=\frac{\omega}{\omega_{1}},
$$

where $\omega$ is the frequency of the plane wave obtained by solving the eigenvalue problem Eq. (14) and $\omega_{1}$ $=\pi^{2} \sqrt{ }\left(E I / \rho d L^{4}\right)$ is the first pinned-pinned flexural resonance frequency of a lattice beam. Consequently, at $\Omega=1$ the cell deformation exhibits the first pinned-pinned flexural mode of the beam.

The point $\mathrm{O}$ corresponds to the long-wavelength limit, where the effective medium representation of the lattice is valid. Two branches of the dispersion curve emanate from the origin $\mathrm{O}$ : these are the longitudinal waves (also known as irrotational or dilatational waves) and transverse waves (also known as distortional, shear, or equivoluminal waves). Recall that the tangent to the dispersion curve at any point gives the group velocity while the secant slope of the line connecting the origin $\mathrm{O}$ to the point of interest on the dispersion curve gives the phase velocity. The two group velocities corresponding to the dispersion branches in the long-wavelength limit correspond to those of an effective elastic medium with modulus as given in Table I for the hexagonal honeycomb. For an isotropic medium with Young's modulus $E^{*}$, shear modulus $G^{*}$, bulk modulus $K^{*}$, and density $\rho^{*}$, the group velocities are given by
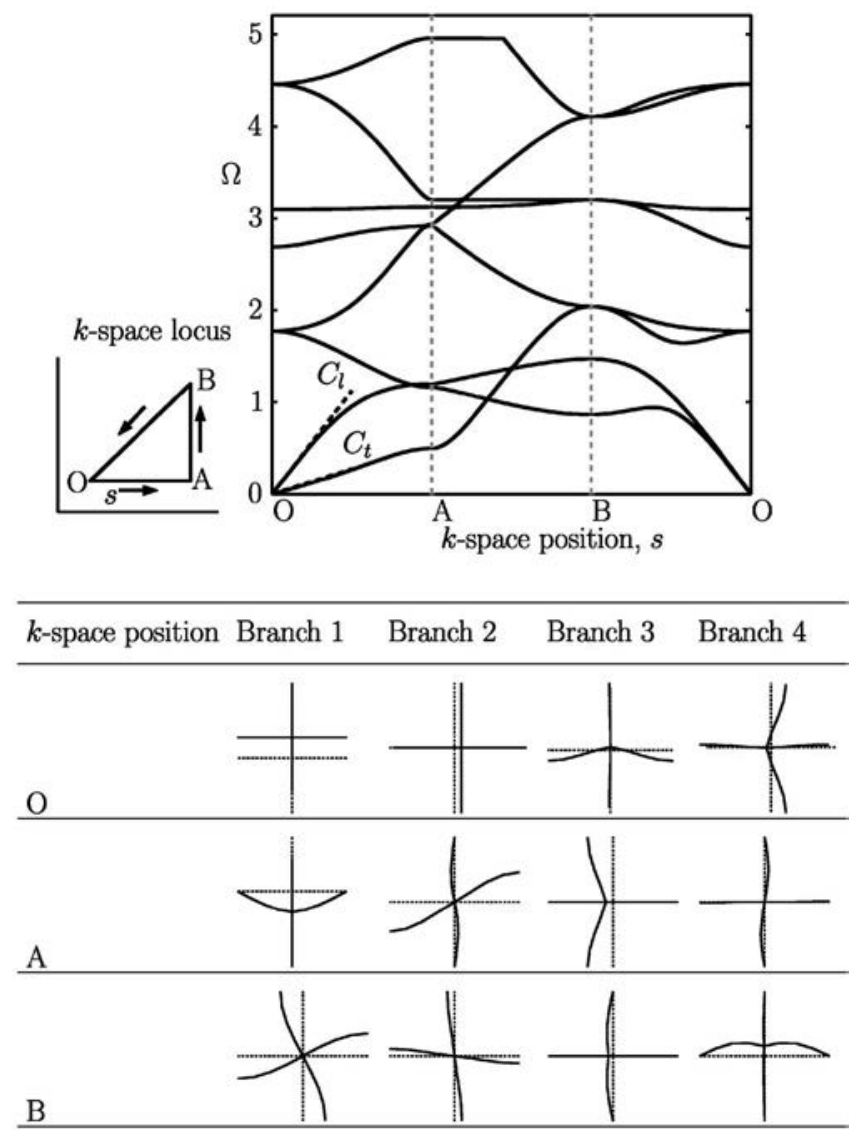

FIG. 13. Band structure of a square honeycomb with a slenderness ratio equal to 10 . The eigenwaves of a typical cell are shown in tabular form. The three rows correspond to the three points $\mathrm{O}, \mathrm{A}$, and $\mathrm{B}$ in $k$ space, while the four columns correspond to the first four dispersion branches in ascending order.

$$
C_{l}=\sqrt{\frac{K^{*}+G^{*}}{\rho^{*}}}, \quad C_{t}=\sqrt{\frac{G^{*}}{\rho^{*}}} .
$$

Thus, the two lines starting at $\mathrm{O}$ and with slopes corresponding to the group velocities of $C_{l}$ and $C_{t}$ are a best approximation to the dispersion curves in the long-wavelength limit. The deviation of these long-wavelength asymptotes from the dispersion curves indicates the range of validity of effective medium theories: at higher frequencies of wave propagation the effective medium results in Table I do not apply. At these frequencies the detailed geometry of the lattice has a significant influence upon the wave-bearing properties.

A common feature of the dispersion curves for the hexagonal honeycomb (and for the other topologies) is the phenomenon of veering of frequencies (or repulsion of the dispersion branches), where the dispersion curves lay close to one another along the locus $\mathrm{O}-\mathrm{A}-\mathrm{B}-\mathrm{O}$ in $k$ space. This is a ubiquitous feature of eigenvalue problems of weakly coupled systems (Perkins and Mote, Jr., 1986). Such veering can be observed in Fig. 8 between the second and third branches of the dispersion curve along the locus O-A. From the magnified picture of the veering zone, it can be noted that the eigenvalues do not cross but veer away from each other.

The eigenwaves of a typical honeycomb cell for each dispersion curve are summarized in tabular form within Figs. 7 and 8 at the points $\mathrm{O}, \mathrm{A}$ and $\mathrm{B}$ in $k$ space. The three rows 

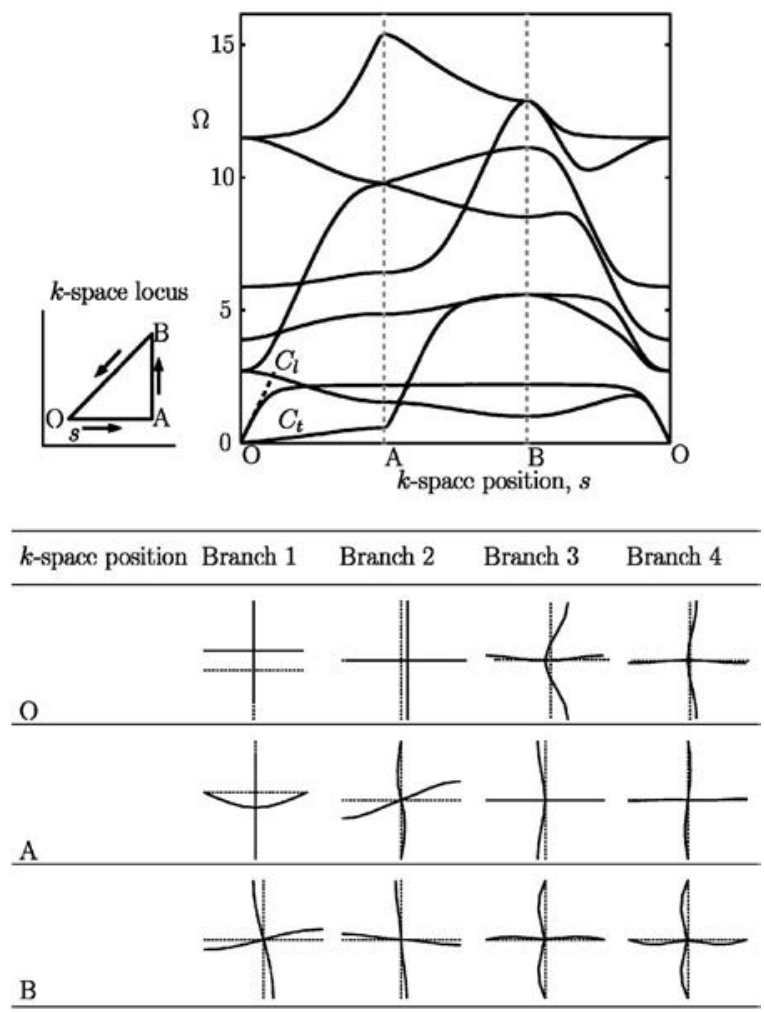

FIG. 14. Band structure of a square honeycomb with slenderness ratio equal to 50. The eigenwaves of a typical cell are shown in tabular form. The three rows correspond to the three points $\mathrm{O}, \mathrm{A}$, and $\mathrm{B}$ in $k$ space, while the four columns correspond to the first four dispersion branches in ascending order.

in each table correspond to the three points $\mathrm{O}, \mathrm{A}$, and $\mathrm{B}$, while the four columns correspond to the first four dispersion branches in ascending order. Consider the four eigenwaves in the first column of Fig. 7. At point $O$, the unit cell exhibits rigid body translation. Transverse wave motion occurs along the first branch, with increasing wave number; the transverse nature of the eigenwaves is clear from the cell deformation at point $\mathrm{A}$. The second column of the table gives the second branch of the dispersion curve and this comprises a longitudinal wave. In higher branches, the eigenwave exhibits combined transverse and longitudinal motion.

The lattice with a slenderness ratio equal to 50 shows a complete bandgap (shaded space) between the sixth and the seventh branches of the dispersion curves. We note that no complete bandgaps are exhibited by the hexagonal honeycomb with a slenderness ratio of 10 (or 20). However, partial bandgaps for confined regions along the locus O-A-B-O are noted in Fig. 7.

\section{B. Kagomé lattice}

The dispersion responses of the Kagomé lattice (and of the triangular honeycomb and square honeycomb) are similarly shown in a plot of $\Omega$ versus length parameter $s$ in $k$ space. See Figs. 9 and 10 for slenderness ratios of 10 and 50, respectively. The long-wavelength asymptotes, as calculated from the group velocities using Eq. (16), are superimposed on the plots and agree with the dispersion curves. The Kagomé lattice does not exhibit any complete bandgaps for the slenderness ratios considered.
It is instructive to compare the long-wavelength behavior of transverse waves in the Kagomé and honeycomb lattices. For the honeycomb, the slope of the dispersion curve associated with the transverse wave decreases with an increase in slenderness ratio while the dispersion curves of the Kagomé lattice do not show this. This can be explained as follows. The transverse wave speed depends upon the ratio of effective shear modulus $G^{*}$ to density according to Eq. (16). Now $G^{*}$ scales as $\rho^{* 3}$ for the hexagonal honeycomb whereas $G^{*}$ scales as $\rho^{*}$ for the Kagomé lattice. Consequently, the transverse wave decreases with an increase in the slenderness ratio for the hexagonal honeycomb, but not for the Kagomé lattice.

The first dispersion curve constitutes transverse wave motion, while the second curve exhibits longitudinal motion. Higher branches display a combination of both transverse and longitudinal motion.

\section{Triangular lattice}

The dispersion results for the triangular lattice are given in Figs. 11 and 12 for slenderness ratios of 10 and 50, respectively. Again, the long-wavelength asymptotes agree with the dispersion curve. For all slenderness ratios considered (10, 20, and 50), a complete bandgap (shaded region) is present. But the location of the bandgap depends upon the slenderness ratio. For a ratio of 10, the gap exists between the fifth and sixth branches of the dispersion curve at a nondimensional frequency $\Omega$ centered on 2.6. At a slenderness ratio of 20, the gap exists between the sixth and seventh branches at a nondimensional frequency $\Omega$ centered on 4.6 (figure not shown). An increase in a slenderness ratio of 50 leads to a bandgap between the third and fourth branches at a nondimensional frequency centered on 2.4. The width of the bandgap is almost independent of the slenderness ratio, and equals about 0.2 .

\section{Square lattice}

The dispersion results for the square lattice are given in Figs. 13 and 14. The group velocities for the longitudinal and shear waves in the long-wavelength limit are given by

$$
C_{l}=\sqrt{\frac{2 K^{*}}{\rho^{*}}}, \quad C_{t}=\sqrt{\frac{G^{*}}{\rho^{*}}},
$$

where the effective bulk modulus $\left(K^{*}\right)$ and shear modulus $\left(G^{*}\right)$ are given in Table I. As before, the long-wavelength asymptotes as calculated from the group velocities via Eq. (17) agree well with the dispersion curve.

The general features are similar to that already discussed for the other lattices: the first and second branches comprise shear waves and longitudinal waves, respectively. Pinnedpinned eigenwaves exist at $\Omega=1$. Above this frequency value the dispersion curves tend to cluster. No complete bandgaps are observed over the range of slenderness ratios considered.

\section{E. Directionality of wave propagation}

The directionality of waves indicates the degree to which a medium is isotropic with respect to wave propaga- 
tion. In an isotropic medium no preferred directions exist, and waves propagate equally in all directions. A common technique for displaying directionality is to construct isofrequency contours of the dispersion surface and to plot these contours in a Cartesian reference frame in $k$ space. The physical coordinate system $(x, y)$ has already been introduced for each lattice in Figs. 1-4. The wave vectors $\left(k_{x}, k_{y}\right)$ are aligned with these physical orthonormal vectors, and form the axes of the isofrequency plots shown in Fig. 15 for each lattice at a slenderness ratio of 50. Contours are given for selected nondimensional frequencies $\Omega$.

The relative shapes of the isofrequency contours are compared in Fig. 15 for the four topologies of interest. The symmetries of these contours show the rotational and reflective symmetries of the parent lattices. At low frequencies, the triangular and hexagonal honeycomb and Kagomé lattice are isotropic, whereas the square honeycomb is strongly anisotropic. At high frequencies, the lattice symmetries have a strong influence upon directionality.

\section{CONCLUSIONS}

Floquet-Bloch principles have been used to explore plane wave propagation in several two-dimensional lattices. Dispersion curves are obtained by solving the eigenvalue problem for wave propagation, and the dependence of frequency upon the wave number has been determined in order to reveal the band structure. Three of the lattices are isotropic under static loading, while the fourth (the square honeycomb) is strongly anisotropic under static loading. The nodal connectivity varies from one structure to the next (from 3 to 6 ), and this has a major influence upon the static behavior. In this study we address whether similar differences in response occur under wave loading. The main findings are as follows.

(1) Long-wavelength asymptotes, based upon effective medium theories, agree with the dispersion curves at low frequency. However, at shorter wavelengths these asymptotes diverge from the dispersion curves, thereby indicating the range of validity of effective medium theories.

(2) Complete bandgaps are present for a wider range of relative density of lattice material for the triangular honeycomb than for the hexagonal honeycomb. They exist at frequencies above the first pin-pin resonance frequency for a single bar of the microstructure.

(3) Wave directionality plots at high frequencies show symmetries that are consistent with those of the parent lattice. Hexagonal, Kagomé, and triangular lattices all exhibit six-fold symmetry. They possess an isotropic response in the long-wavelength limit. The square lattice has four-fold symmetry and is strongly anisotropic over the full frequency range.

Imperfections in the lattice topology can lead to the localization of elastic waves, as discussed in Bendiksen (2000) and Hodges and Woodhouse (1983). While there is significant literature dealing with the localization of elastic waves in one-dimensional periodic structures, little is known about two-dimensional periodic structures. Future work will address the influence of imperfections on the band structure of
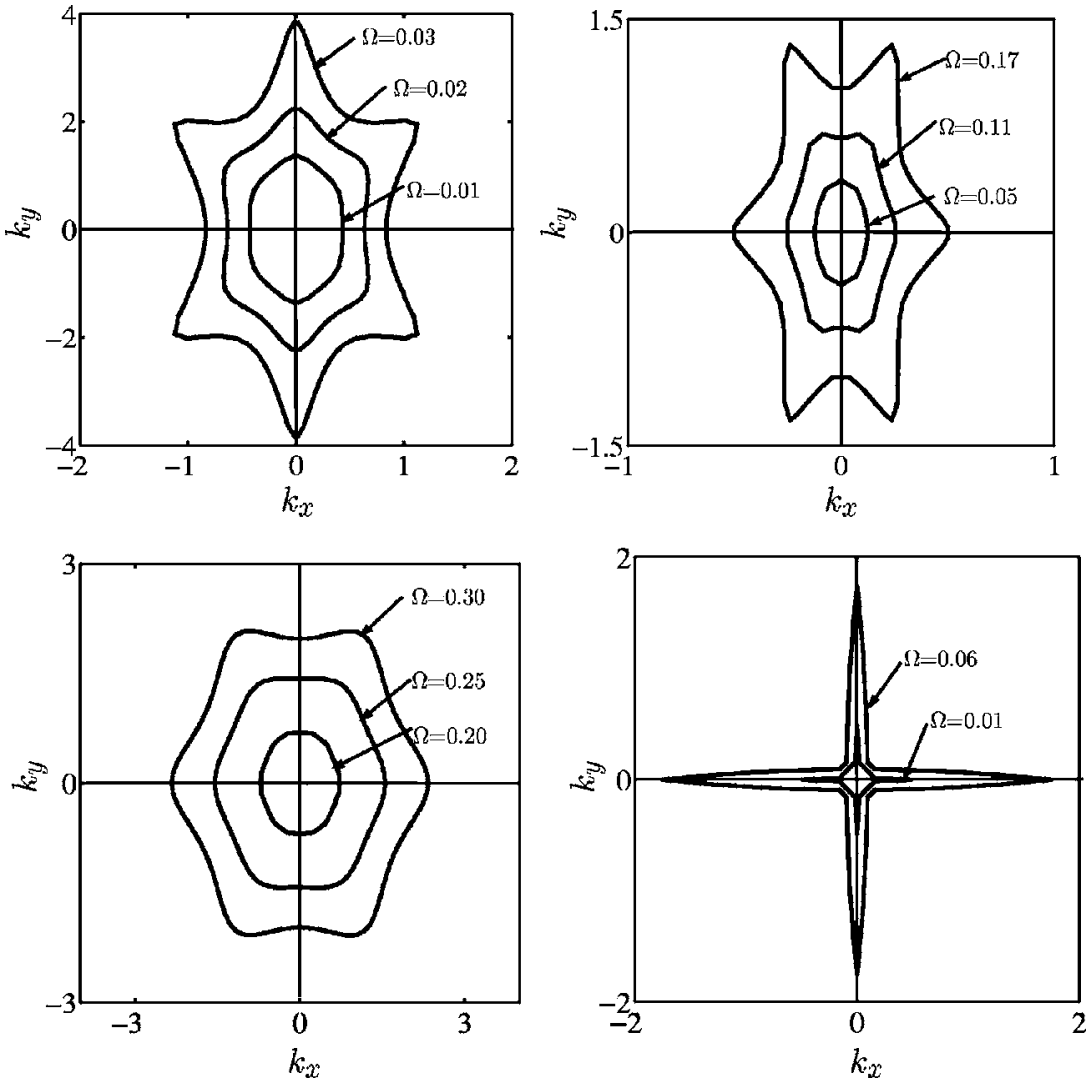

FIG. 15. Directionality of plane wave propagation in the four lattice topologies with slenderness ratio equal to 50: (a) hexagonal honeycomb; (b) Kagomé lattice; (c) triangular honeycomb; (d) square honeycomb. The nondimensional frequency $(\Omega)$ associated with each contour is labeled. At each frequency, energy flow direction is given by the normal to the contour and is in the direction of maximum rate of change of frequencies. 
the topologies studied in this work. It is anticipated that a small imperfection is sufficient to alter significantly the band structure.

\section{ACKNOWLEDGMENTS}

The work carried out in this report is partially funded by the EU-HYMM project: 6th FP-NMP STREP-Contract No: NMP3-CT-2003-505206. Helpful technical discussions with R. S. Langley and Angelo Merlo are gratefully acknowledged.

\section{APPENDIX: SHAPE FUNCTIONS OF TIMOSHENKO BEAM-COLUMN ELEMENT}

The shape functions $a_{r}, b_{r}, c_{r}, r=1 \cdots 6$ for the six nodal displacements of a beam-column element shown in Fig. 5 are as follows:

$$
\begin{aligned}
& a_{1}=\frac{1}{2}(1-\xi), \\
& a_{2}=0, \quad a_{3}=0, \\
& a_{4}=1 / 2(1+\xi), \\
& a_{5}=0, \quad a_{6}=0, \\
& b_{1}=0 \text {, } \\
& b_{2}=\frac{1}{4(1+3 \beta)}\left[2+6 \beta-3(1+2 \beta) \xi+\xi^{3}\right] \text {, } \\
& b_{3}=\frac{1}{4(1+3 \beta)}\left[1+3 \beta-\xi-(1+3 \beta) \xi^{2}+\xi^{3}\right], \\
& b_{4}=0 \text {, } \\
& b_{5}=\frac{1}{4(1+3 \beta)}\left[2+6 \beta+3(1+2 \beta) \xi-\xi^{3}\right] \text {, } \\
& b_{6}=\frac{1}{4(1+3 \beta)}\left[-(1+3 \beta)-\xi+(1+3 \beta) \beta^{2}+\xi^{3}\right] \text {, } \\
& c_{1}=0 \text {, } \\
& c_{2}=\frac{1}{4(1+3 \beta)}\left(-3+3 \xi^{2}\right) \\
& c_{3}=\frac{1}{4(1+3 \beta)}\left[-1+6 \beta-(2+6 \beta) \xi+3 \xi^{2}\right],
\end{aligned}
$$

$$
\begin{aligned}
& c_{4}=0, \\
& c_{5}=\frac{1}{4(1+3 \beta)}\left(3-3 \xi^{2}\right), \\
& c_{6}=\frac{1}{4(1+3 \beta)}\left[-1+6 \beta+(2+6 \beta) \xi+3 \xi^{2}\right],
\end{aligned}
$$

where

$$
\xi=\frac{x}{L}, \quad \beta=\frac{E I_{z}}{\kappa G A L^{2}} .
$$

In the above equation, $E$ and $G$ denote the Young's modulus and rigidity modulus respectively; $L, A, I_{z}$ denote the length, cross-sectional area and second moment of area of the beam, respectively; and $\kappa$ denotes the shear correction factor used in Timoshenko beam theory (Weaver and Jonhston, 1987).

Bendiksen, O. O. (2000). "Localization phenomenon in structural dynamics," Chaos, Solitons Fractals 11, 1621-1660.

Brillouin, L. (1953). Wave Propagation in Periodic Structures, 2nd ed. (Dover, New York).

Christensen, R. M. (2000). "Mechanics of cellular and other low density materials," Int. J. Solids Struct. 37, 93-104.

Gibson, L. J., and Ashby, M. F. (1997). Cellular Solids: Structure and Properties, 2nd ed. (Cambridge University Press, Cambridge).

Hodges, C. H., and Woodhouse, J. (1983). "Vibration isolation from irregularity in a nearly periodic structure: theory and measurements," J. Acoust. Soc. Am. 74, 894-905.

Hutchinson, R. G. (2004). "Mechanics of lattice materials," Ph.D. thesis, University of Cambridge, Department of Engineering.

Kittel, C. (1962). Elementary Solid State Physics: A Short Course, 1st ed. (Wiley, New York).

Langley, R. S. (1993). "A note on the forced boundary conditions for twodimensional periodic structures with corner freedoms," J. Sound Vib. 167, 377-381.

Langley, R. S., Bardell, N. S., and Ruivo, H. M. (1997). "The response of two-dimensional periodic structures to harmonic point loading: a theoretical and experimental study of a beam grillage," J. Sound Vib. 207, 521535.

Mead, D. (1973). "A general theory of harmonic wave propagation in linear periodic systems with multiple coupling," J. Sound Vib. 27, 235-260.

Mead, D. (1996). "Wave propagation in continuous periodic structures: research contributions from Southampton 1964-1995," J. Sound Vib. 190, 495-524.

Noor, A. K., Anderson, M. S., and Greene, W. H. (1978). "Continuum models for beam-and platelike lattice structures," AIAA J. 16, 1219-1228.

Perkins, N. C., and Mote, C. D. Jr. (1986). "Comment on curve veering in eigenvalue problems," J. Sound Vib. 106, 451-463.

Ruzzene, M., Scarpa, F., and Soranna, F. (2003). "Wave beaming effects in two-dimensional cellular structures," Smart Mater. Struct. 12, 363-372.

Sigmund, O., and Jensen, J. S. (2003). "Systematic design of photonic bandgap materials and structures by topology optimization," Proc. R. Soc. London, Ser. A 361, 1001-1019.

Torquato, S., Gubiansky, L. V., Silva, M. J., and Gibson, L. J. (1998). "Effective mechanical and transport properties of cellular solids," Int. J. Mech. Sci. 40, 71-82.

Weaver, W., and Jonhston, P. R. (1987). Structural Dynamics by Finite Elements, 1st ed. (Prentice-Hall, Englewood Cliffs, NJ).

Wicks, N., and Hutchinson, J. W. (2001). "Optimal truss plates," Int. J. Solids Struct. 38, 5165-5183. 